\title{
Necessary condition analysis in tourism research
}

\section{Introduction}

Necessary condition analysis (abbreviated as NCA) is a data analysis method based on the causal logic of necessity: if the necessary condition is absent, then the outcome will not occur. This approach offers valuable insights into whether and the extent to which certain conditions are necessary for the outcome of interest, which is different from the underlying sufficiency interpretations of regression and fuzzy set qualitative comparative analysis (QCA). With the growth of NCA applications across scientific domains (Dul, 2016a; Dul et al., forthcoming; Vis \& Dul, 2018, Karwowski et al., 2016; Van der Valk et al., 2016), there is also potential for novel applications in tourism.

This research note presents information for scholars seeking to understand the potential applicability of NCA in tourism, as well as guidelines for appropriate application and interpretation of results. The note provides useful details on the underlying logic, the formulation of the necessary condition hypothesis, core steps of the analysis, and interpretation of results with the help of an illustrative case. Researchers wanting to learn more about the approach should consult Dul (2016a \& 2019) and download the free NCA software package in R through https://CRAN.R-project.org/package=NCA.

\section{Early attempts of NCA applications in tourism research}

In the first application of NCA in tourism research, Lee and Jeong (2019) studied the necessary nature of certain hedonic elements (positive affect, negative affect and carefreeness) on tourists' eudaimonic (well-being related) experiences. Olya and colleagues (Gannon et al. 2019; Olya \& Al-ansi 2018; Olya et al. 2019) have used QCA, which has a different approach to necessity than the approach advocated in NCA. Two main differences are that QCA uses set membership scores and Boolean algebra, and consequently can make statements of in-kind necessity ("the presence (or absence) of X is necessary for the presence (or absence) of Y"), whereas NCA uses variable scores and linear algebra to allow for making in-degree statements of necessity ("level A of $\mathrm{X}$ is necessary for level B of Y"). A more elaborate discussion about these differences can be found in Dul (2016b) and Vis and Dul (2018).

In the following sections we outline the building blocks that researchers in tourism and beyond the sector can utilise. For demonstration purposes we draw on an example from recent tourism research by Liu et al. (2019) that explains how tourists' perceived value of the overall experience is influenced by supplier innovations in the tourism value chain.

\section{Underlying logic and the formulation of the necessary condition hypothesis}

A necessary condition must be present for obtaining the outcome, but the presence of the condition is not automatically sufficient to achieve it. In other words, without the necessary condition, the absence of the outcome is guaranteed. The underlying causal logic of necessary conditions is different from sufficiency-based causal reasoning. Necessary conditions are bottlenecks that can stop the outcome from occurring. However, common thinking about causal 
relationships typically is sufficiency-based. For instance, regression analysis results are interpreted as various conditions that jointly help to produce the outcome. Whereas necessary conditions work in isolation, regression results are based on additive logic assuming that conditions can compensate for each other.

Theoretical 'necessary but not sufficient' statements that are commonly formulated in literature indicate a good potential for necessary condition hypothesis. Also, traditional sufficiency-type hypotheses may be reformulated into necessary condition hypotheses, when there is theoretical justification for the condition to be a necessary cause. Alternatively, the necessary condition hypothesis may be formulated based on theoretical framing and substantial contextual understanding, even in the absence of previous empirical investigation. A tourism example for the reformulation of conventional sufficiency-type hypothesis towards necessary condition hypothesis is as follows:

Sufficiency-type hypothesis: Convenience increases tourists' Perceived utility. Necessary condition hypothesis: Convenience is necessary for tourists' Perceived utility.

The sufficiency-type hypothesis proposes that Convenience increases Perceived utility, i.e. Convenience is sufficient for increasing the outcome. Without Convenience, Perceived utility may decrease, but there are other conditions through which the outcome can be achieved. For example, immersion, learning, and surprise could also increase Perceived utility and make up for the lack of Convenience.

However, the necessary condition hypothesis suggests Convenience is a bottleneck that cannot be compensated by other conditions when it is absent. Convenience then not only increases the Perceived utility but in fact enables it. Without (a high level of) Convenience, the lack of (or low level of) Perceived utility is guaranteed. In the following section we outline how to test the necessary condition hypothesis.

\section{Applying and presenting NCA: Scatterplot, effect size, and the bottleneck table}

The analysis for testing a necessary condition hypothesis should have at least three pillars, namely the presentation of the scatterplot, the effect size and the bottleneck table. First, a starting point is to examine the scatterplot of sample data, which shows the potential necessary condition X (horizontal) against the outcome $\mathrm{Y}$ (vertical). If there is an 'empty' space in the upper left corner, the presence (or high level) of $\mathrm{X}$ may be a necessary condition for the presence (or high level) of Y. In Figure 1, there appears to be an 'empty' space in the upper left corner of the scatterplot of Convenience and Perceived utility. The line through the middle of the observations is the ordinary least squares (OLS) regression line. Two alternative 'ceiling lines' are shown on top of the data: the step function called Ceiling Envelopment - Free Disposal Hull (CE-FDH), which is commonly used for discrete data, and a trend line through the upper left corner point of this line, called the Ceiling Regression - Free Disposal Hull (CRFDH), which is commonly used for continuous data or when a linear ceiling is assumed. A ceiling line defines the size of the 'empty' space.

Secondly, one should assess whether the necessary condition is large enough to be taken seriously. Thus, there is a need to produce an effect size measure (d). The effect size represents how much a given value of the necessary condition $\mathrm{X}$ constrains $\mathrm{Y}$ (i.e., the size of the 
constraint that the ceiling poses on the outcome). The effect (constraint) is stronger when the ceiling zone ('empty' space) is larger. Hence, the effect size of a necessary condition is based on the size of the ceiling zone compared to the size of the entire area that can contain observations given the extreme values of the condition and the outcome. As a guideline, researchers can consider $0<\mathrm{d}<0.1$ as 'small effect', $0.1 \leq \mathrm{d}<0.3$ as 'medium effect', $0.3 \leq \mathrm{d}$ $<0.5$ as 'large effect', and $d \geq 0.5$ as 'very large effect'.

In the illustrative case $\mathrm{d}=0.108$ for CR-FDH (for CE-FDH it is $\mathrm{d}=0.121$ ). With medium effect size, the Convenience condition poses some constraint on Perceived utility. Because 'empty' spaces can be due to chance when $\mathrm{X}$ and $\mathrm{Y}$ are unrelated, a $\mathrm{p}$ value is produced to check for this randomness, similar to other null hypothesis tests (Dul et al. 2019). In the current example, the $\mathrm{p}$ value of the observed effect size is $\mathrm{p}<0.001$ for this space produced by CR-FDH (and 0.002 for the 'empty' space produced by CE-FDH).

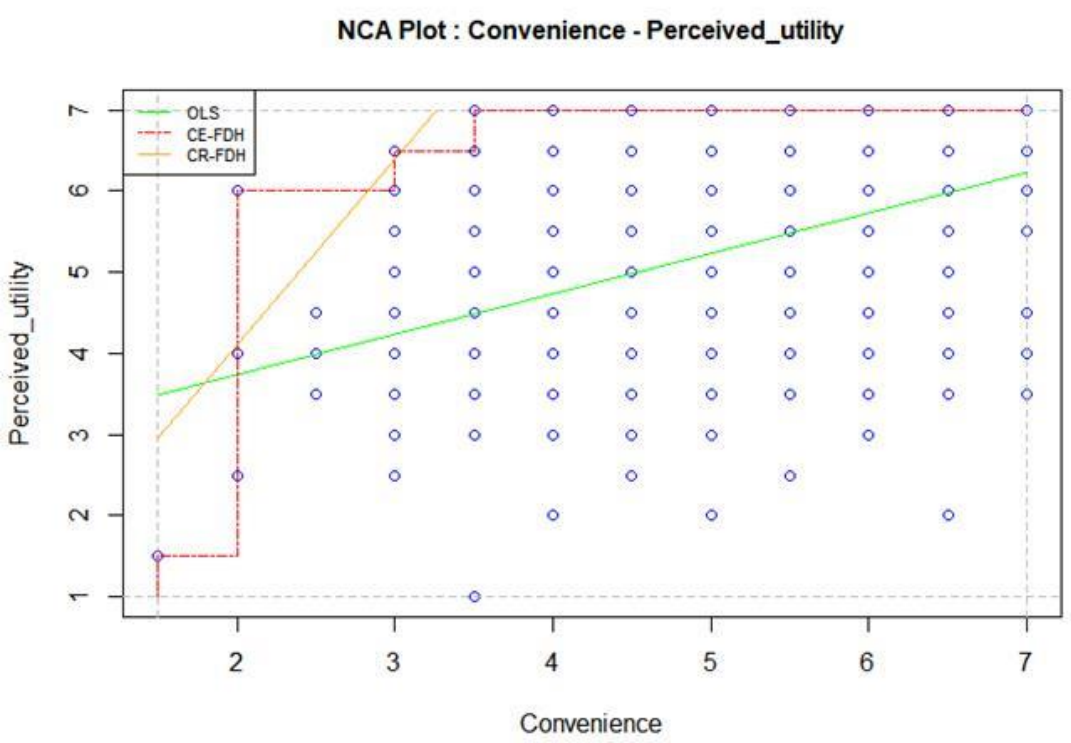

Figure 1. Scatterplot of Convenience against Perceived utility

Finally, the bottleneck table, which is particularly interesting in situations with multiple necessary conditions, shows which levels of the conditions are necessary to achieve certain levels of the outcome. Assuming a linear ceiling relation between the two variables the values presented in the bottleneck table are based on the CR-FDH ceiling line (Table 1.). For demonstration purposes, the levels of Convenience are shown below for achieving different levels of Perceived utility. 

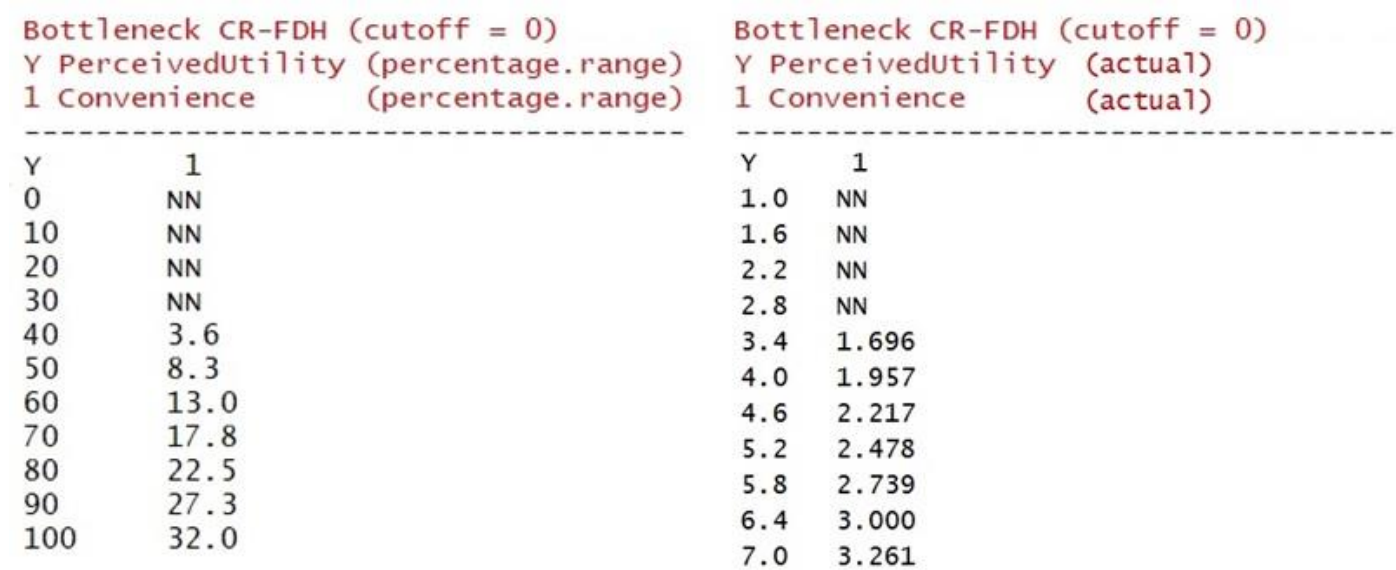

Table 1. Bottleneck tables for Convenience as a necessary condition for Perceived utility, using the CR-FDH ceiling line. Left: percentage range, e.g. for achieving $50 \%$ of observed range of Perceived utility $(0 \%=$ score 1 , $100 \%=$ score 7$), 8.3 \%$ of observed range of Convenience $(0 \%=$ score $1.5,100 \%=$ score 7$)$ is necessary. Right: actual values, e.g. for achieving score 4 Perceived utility, a score of 2 (1.957) Convenience is necessary.

To check how specific levels of the outcome condition can be achieved, the bottleneck table represents the ceiling line and can be read row by row. For instance, Table 1 (Left) shows that to reach low level Perceived utility (30\% or less), Convenience is not necessary (NN). To reach medium level Perceived utility (50\%), at least $8.3 \%$ Convenience is the minimum requirement, while for top level Perceived utility (100\%) at least $32 \%$ Convenience is required. Hence, when the tourism suppliers aim to reach medium to high Perceived utility, ensuring some - but not necessarily excessive levels of - Convenience is necessary. If this level of Convenience is not in place, the outcome will not occur and spending resources on other factors than the bottleneck factor is a waste. The bottleneck table can help identify combinations of necessary conditions and their required levels when assessing the necessity of multiple conditions for achieving the outcome.

\section{Future NCA applications in tourism}

Future applications could address, for example, the necessity of previous experiences and novelty seeking for tourist satisfaction and loyalty, and the extent to which non-compensatory preferences, such as safety (Koo et al., 2019) are necessary for destination choice. Contributions can be formulated by confirming or falsifying existing theories, as well as the identification of necessary conditions that have not been explored previously. The NCA approach enables the study of in-degree necessity, i.e. it helps to identify the levels of conditions that are necessary for reaching certain levels of the outcome. This differs from the study of in-kind necessity, i.e. whether the conditions are necessary for the outcome of interest (Vis \& Dul, 2018). In addition, NCA can be applied either solely or in combination with other methods, depending on the aims of the specific research project. However, clear links should always be made to the underlying necessity logic, along with the formulation of necessary condition hypotheses and presentation of the core elements of the analysis: the scatterplot, effect size and the bottleneck table. 


\section{References}

Dul, J. (2016a). Necessary condition analysis (NCA) logic and methodology of "necessary but not sufficient" causality. Organizational Research Methods, 19(1), 10-52.

Dul, J. (2016b). Identifying single necessary conditions with NCA and fsQCA. Journal of Business Research, 69(4), 1516-1523.

Dul, J. (2019). Conducting necessary condition analysis. London: Sage Publications.

Dul, J., van der Laan, E., \& Kuik, R. A statistical significance test for Necessary Condition Analysis. Organizational Research Methods, 1094428118795272, forthcoming

Gannon, M., Taheri, B., \& Olya, H. (2019). Festival quality, self-connection, and bragging. Annals of Tourism Research, 76, 239-252.

Karwowski, M., Dul, J., Gralewski, J., Jauk, E., Jankowska, D.M., Gajda, A., Chruszczewski, M.H., \& Benedek, M. (2016). Is creativity without intelligence possible? A necessary condition analysis. Intelligence, 57, 105-117.

Koo, T., Andrew, T., Ann, W., \& Caponecchia, C. (2019). How safety risk information and alternative forms of presenting it affect traveller decision rules in international flight choice. Journal of Travel Research, 58(3), 480-495.

Lee, W., \& Jeong, C. (2019). Beyond the correlation between tourist eudaimonic and hedonic experiences: necessary condition analysis. Current Issues in Tourism, forthcoming.

Liu, Y., Li. C., McCabe, S., \& Xu, H. (2019). How small things affect the big picture? The effect of service product innovation on perceived experience value. International Journal of Contemporary Hospitality Management, forthcoming.

Olya, H.G., \& Al-ansi, A. (2018). Risk assessment of halal products and services: Implication for tourism industry. Tourism Management, 65, 279-291.

Olya, H.G., Bagheri, P., \& Tümer, M. (2019). Decoding behavioural responses of green hotel guests: A deeper insight into the application of the theory of planned behaviour. International Journal of Contemporary Hospitality Management, forthcoming.

Van der Valk, W., Sumo, R., Dul, J., \& Schroeder, R.G. (2016). When are contracts and trust necessary for innovation in buyer-supplier relationships? A necessary condition analysis. Journal of Purchasing and Supply Management, 22(4), 266-277.

Vis, B., \& Dul, J. (2018). Analyzing relationships of necessity not just in kind but also in degree: Complementing fsQCA with NCA. Sociological Methods \& Research, 47(4), 872-899. 